\title{
Tripterine Restrains the Aggressiveness of Hepatocellular Carcinoma Cell via Regulating miRNA-532-5p/CXCL2 Axis
}

This article was published in the following Dove Press journal: OncoTargets and Therapy

\author{
Zhi Tao Jiang ${ }^{1, *}$ \\ Yi Han',* \\ Xiao Yan Liu' \\ Ling Yan Lv' \\ Jin Huo Pan ${ }^{2}$ \\ Chun Di Liu' \\ 'Department of Pharmacy Office, \\ Zhangjiagang Hospital of Traditional \\ Chinese Medicine Affiliated to Nanjing \\ University of Chinese Medicine, \\ Zhangjiagang, Jiangsu, People's Republic of \\ China; ${ }^{2}$ College of Pharmacy, Nanjing \\ University of Chinese Medicine, Nanjing, \\ Jiangsu, People's Republic of China
}

*These authors contributed equally to this work
Correspondence: Jin Huo Pan

Nanjing University of Chinese Medicine, Nanjing, Jiangsu, People's Republic of China

Email panjinhuonj@I63.com

Chun Di Liu

Zhangjiagang Hospital of Traditional

Chinese Medicine Affiliated to Nanjing

University of Chinese Medicine,

Zhangjiagang, Jiangsu, People's Republic of

China

Email liucd0723@foxmail.com
Introduction: Triterpene has attracted considerable interests because it exhibits anticancer effects. However, the effects of tripterine on hepatocellular carcinoma (HCC) are not well studied. In the current study, the mechanism of tripterine on HCC cells growth and metastasis was examined.

Methods: The inhibitory effect on the growth and aggressiveness in HCC cells was analyzed by Cell Counting Kit-8 (CCK-8), wound healing and Transwell assay. The levels of microRNA-532-5p (miR-532-5p) in HCC cells and tissues were measured using qRTPCR. The expression of chemokine (C-X-C Motif) ligand 2 (CXCL2) was determined by Western blotting and immunohistochemistry (IHC). Luciferase reporter gene assay was used to validate the binding between miR-532-5p and CXCL2. The impact of tripterine on the growth and metastasis of HCC cells in vivo was analyzed using transplanted tumor model and experimental lung metastasis model, respectively.

Results: We found that tripterine inhibited HCC cells proliferation, migration ability and invasion. Under tripterine treatment, the level of miR-532-5p was strikingly raised, and overexpression of miR-532-5p reduced cell viability and metastatic-related traits. In addition, we identified CXCL2 as a target of miR-532-5p in HCC. Rescue experiments indicated that overexpression of CXCL2 restored the migration and invasive capacity of HCC cells inhibited by miR-532-5p or tripterine treatment. Finally, the tumor growth and metastatic ability of $\mathrm{HCC}$ MHCC $97 \mathrm{H}$ cell in vivo were also significantly restrained by tripterine. The expression of CXCL2 was distinctly decreased and miR-532-5p level was increased by tripterine in vivo.

Conclusion: In conclusion, tripterine inhibits the growth, migration ability and invasiveness of HCC cells through intervening miR-532-5p/CXCL2.

Keywords: tripterine, HCC, miRNA-532-5p, CXCL2, Metastasis

\section{Introduction}

Hepatocellular carcinoma (HCC) is still the commonest liver cancer and is the mainly cause of cancer-associated deaths worldwide. ${ }^{1}$ In the last decades, surgical resection, liver transplantation, transarterial chemoembolization (TACE) and targeted agents substantially improved the clinical outcomes of patients with HCC. ${ }^{2}$ Nevertheless, high recurrence rate and distant metastasis in HCC patient after surgery result in an unsatisfied overall survival. ${ }^{3}$ Hence, investigation of the molecular mechanisms involved into HCC metastasis may be essential to improve the overall survival. 
Recently, the Traditional Chinese Medicine (TCM) has become a "hot spot" for its abilities to simultaneously address multiple targets, no side-effects and improving the sensitivity of chemotherapy or radiation therapy. Tripterine is a natural compound, which is mainly derived from Tripterygium wilfordii. Tripterine has been proved to exhibit antiinflammatory, antioxidant, anti-cancer and anti-fibrotic activities. ${ }^{4-8}$ In clinic, tripterine has been used to treat rheumatoid arthritis, leprosy reaction and other autoimmune diseases. ${ }^{9,10}$ Besides, tripterine regulates cell survival and inflammation through regulating Nuclear Factor Kappa $\mathrm{B}(\mathrm{NF}-\mathrm{kB})$, Janus Kinase/Signal Transducer and Activator of Transcription (JAK/STAT), Mitogen-Activated Protein Kinase (MAPK) and Phosphatidylinositol-4,5-Bisphosphate 3-Kinase-Threonine> Kinase (PI3K-AKT) pathway., ${ }^{9,10}$ Previous investigations have indicated that tripterine inhibits angiogenesis-mediated tumor growth through inhibiting AKT/mammalian target of rapamycin (mTOR) pathway. ${ }^{11}$ Nevertheless, the anti-liver cancer functions of tripterine have not been illuminated well, and the underlying mechanisms are still a mystery.

Plenty of investigations indicate that microRNAs (miRNAs) are commonly deregulated in cancers and modulate cancer cell growth and metastasis. For example, miR-143-3p acts as a suppressor through interfering cell growth, epithelialmesenchymal transition (EMT) and metastasis via modulating RNA-binding protein quaking 5 (QKI-5) in human esophageal squamous cell carcinoma (ESCC). ${ }^{12}$ MiR-140-5p represses the growth, migration and invasiveness of gastric carcinoma cell by modulating YES Proto-Oncogene 1 (YES1). ${ }^{13}$ Recently, miR-532-5p has been found that inhibit renal cancer cell growth through disrupting the ETS Proto-Oncogene 1 (ETS1)-mediated positive feedback loop with the KRAS Proto-Oncogene/Nucleosome Assembly Protein 1 Like 1/Mitogen-Activated Protein Kinase 1 (KRAS/NAP1L1/ MAPK1) axis. ${ }^{14}$ In addition, miR-532-5p silencing induces HCC cells proliferation and metastatic capacity in vitro by influencing CXCL2 expression. ${ }^{15}$

In our research, we investigated the role of tripterine on the growth and metastasis suppression of HCC cells. Microarray analysis revealed that the expression of miR532-5p was substantially raised upon tripterine treatment in HCC cells. Notably, tripterine-induced growth, migration and invasion inhibition was partly dependent on the decrease of CXCL2, which was the downstream target gene of miR$532-5 \mathrm{p}$. Altogether, our findings reveal a novel function of tripterine as the miR-532-5p-CXCL2 axis inhibitor to restrain $\mathrm{HCC}$ tumorigenesis and metastasis.

\section{Materials and Methods \\ Cell Culture}

HCC cell lines (HCCLM3, HepG2, SMMC771 and MHCC97H) and normal human hepatic cell line LO2 cells were obtained from the Stem Cell Bank, Chinese Academy of Sciences (Shanghai, China). Cells were maintained in Dulbecco's Modified Eagle Medium (DMEM) (Thermo Fisher Scientific, Waltham, MA, USA) with 10\% fetal bovine serum (FBS) (Thermo Fisher Scientific) and supplemented with $1 \%$ penicillin/streptomycin (Thermo Fisher Scientific). Cells lines were maintained in a humidified atmosphere containing $5 \% \mathrm{CO}_{2}$ at $37^{\circ} \mathrm{C}$. Tripterine (purity $\geq 98 \%$ ) was bought from National Institutes for Food and Drug Control (cat \# 111946-201501, China). Tripterine was dissolved in dimethyl sulfoxide (DMSO, Sigma-Aldrich, Shanghai, China) and made up with the culture medium so that the concentration of DMSO was $0.1 \%$.

\section{HCC Tissues}

Forty-six paired HCC biopsy samples and corresponding non-cancerous liver biopsy tissues were collected at the Zhangjiagang Hospital of Traditional Chinese Medicine affiliated to Nanjing University of Chinese Medicine. Exclusion criteria were an age of below 18 years, a history of another malignant disease within the last five years and a history of organ transplantation. The pathological judgment was determined by two senior pathologists with double-blind. None of preoperative treatment was done. The samples were rapidly frozen and stored in liquid nitrogen. This study was approved by the Ethics Committee of Zhangjiagang Hospital of Traditional Chinese Medicine affiliated to Nanjing University of Chinese Medicine and conforms to the Code of Ethics of the World Medical Association (Declaration of Helsinki) printed in the British Medical Journal (18 July 1964). The written informed consent was obtained from all participants who were involved in the study. The clinical features of all patients were summarized in Supplementary Table 1.

\section{Cell Transfections}

MiRNA negative control (miR-NC), miR-532-5p mimics and miR-532-5p inhibitor were obtained from Nanjing KeyGen Biotech. Inc. (Nanjing, Jiangsu, China). The CXCL2 pcDNA3.1 expression vector (pc-CXCL2) and pcDNA3.1 empty vector was purchased from Miaoling Biotech (cat \# P0157, Wuhan, Hubei, China). MHCC97H and HCCLM3 cells were transfected with miR-532-5p or 
pc-CXCL2 using Lipofectamine 3000 (cat \# L3000015, Thermo Fisher Scientific) according to the instructions.

\section{Cell Proliferation Assay}

Briefly, HCCLM3 or MHCC97H cells $\left(5 \times 10^{3}\right)$ were seeded into 96 -well plates and treated by $0,0.1,0.3,1,3$, or $5 \mu \mathrm{M}$. After culturing the cells for $48 \mathrm{hrs}, 10 \mu \mathrm{L}$ of Cell Counting Kit-8 (CCK-8; Dojindo, Beijing, China) was added into 96well plates. After $30 \mathrm{~min}$, the absorbance at each time point was measured at $450 \mathrm{~nm}$. IC50 was calculated. HCCLM3 or MHCC97H cells $\left(5 \times 10^{3}\right)$ were cultured into 96-well plates. After $24 \mathrm{hrs}$, cells were cultured using media containing tripterine $(0.3 \mu \mathrm{M}, 1 \mu \mathrm{M}$ or $3 \mu \mathrm{M})$. After culturing the cells for $24 \mathrm{hrs}$ or $48 \mathrm{hrs}, 10 \mu \mathrm{L}$ of CCK-8 solution was added into 96-well plates. After $30 \mathrm{~min}$, the absorbance at each time point was measured at $450 \mathrm{~nm}$.

\section{Wound-Healing Assay}

HCCLM3 or MHCC97H cells $\left(5 \times 10^{5}\right)$ were maintained into six-well plates. After cells reached $100 \%$ confluence, a straight scratch was made using a $200 \mu \mathrm{L}$ pipette tip. After washing, cells were treated with tripterine for $24 \mathrm{hrs}$. The images of wound healing were recorded at $0 \mathrm{hr}$ or $24 \mathrm{hrs}$, respectively.

\section{Transwell Invasion}

Transwell invasion chamber $(8.0 \mu \mathrm{m}$, Corning Costar, Corning, NY, USA) was coated with Matrigel (Corning). HCCLM3 or MHCC97H cells $\left(5 \times 10^{3}\right)$ were seeded into the upper chamber with FBS-free media containing tripterine $(0.3 \mu \mathrm{M}, 1 \mu \mathrm{M}$ or $3 \mu \mathrm{M})$. The lower chamber of Transwell was filled with $600 \mu \mathrm{L}$ of medium supplemented with $20 \%$ FBS. After 24 hrs, the invaded HCC cells were fixed by $4 \%$ paraformaldehyde and then stained using $1 \%$ crystal violet.

\section{Colony Formation}

HCCLM3 or MHCC97H cells $\left(2 \times 10^{3}\right)$ were cultured into 6 -well plates. After $24 \mathrm{hrs}$, cells were cultured under tripterine $(0.3 \mu \mathrm{M}, 1 \mu \mathrm{M}$ or $3 \mu \mathrm{M})$. After 14 days, the cells were fixed with 4\% Polyoxymethylene and stained using Giemsa Stain (Abcam, Cambridge, UK). Afterwards, the cell colonies were counted directly with the naked eyes.

\section{Microarray Analysis}

MiRNAs were extracted from HCCLM3 and MHCC97H cells treated with or without tripterine using a miRNeasy Mini Kit (cat \# 217004, Qiagen, Valencia, CA, USA) according to the instructions. cDNA was prepared by using an All-in-one miRNA First Strand cDNA Synthesis Kit (cat \# QP007, GeneCopoeia, Guangzhou, China). A miProfile ${ }^{\mathrm{TM}}$ human cancer miRNA qPCR array (cat \# QM004, GeneCopoeia) was applied for miRNAs expression profile analysis. An All-in-One ${ }^{\mathrm{TM}}$ miRNA qPCR Detection Kit (cat \# QP016, GeneCopoeia) was utilized for real-time PCR reactions on the miRNA PCR Array and the real-time instrument was an ABI 7500 Fast (Applied Biosystems, USA).

\section{Quantitative Real-Time PCR (qRT-PCR) Assay}

RNAs were extracted from HCCLM3 or MHCC97H cells using TRIzol (Thermo Fisher Scientific) and subjected to reverse transcription using a TakaRa PrimeScript ${ }^{\text {TM }}$ RT kit (cat \# RR055B, Takara, Dalian, China). qRT-PCR assay was carried out using a SYBR ${ }^{\circledR}$ Premix Ex Taq ${ }^{\mathrm{TM}}$ II (Takara) in a CFX96 Touch $^{\mathrm{TM}}$ real-time PCR detection system (Bio-Rad Laboratories, Hercules, CA, USA). MiRNAs were extracted using the Qiagen miRNeasy Mini Kit (cat \# 217004, Qiagen) according to the manufacturer's protocol. The level of miR532-5p was detected using a TaqMan miRNA assay (Applied Biosystems, Foster City, CA, USA). Primer sequences were as following. ${ }^{16-19}$ CXCL2: forward, 5'-CCAACCACCAGGCT ACAGG-3'; reverse, 5'-GCGTCACACTCAAGCTCTG-3'; U6: forward, 5'-ATTGGAACGATACAGAGAAGATT-3'; reverse, 5'-GGAACGCTTCACGAATTTG-3'; miR-532-5p: forward, 5'-CATGCCTTGAGTGTAGGACTGT-3'; reverse, 5'-CATGGCCCTGAAATACGAAGTC-3'; GAPDH: forward 5'-ACCCACTCCTCCACCTTTGAC-3'; reverse, 5'-TGTTG CTGTAGCCAAATTCGTT-3'. U6 was used as an internal control for normalizing the miRNA-532-5p expression level. GAPDH was used as an internal control for normalizing the CXCL2 expression level. Candidate reference genes for the normalization and the expression stability were calculated by the NormFinder program. ${ }^{20,21}$ The PCR amplification conditions were: $50^{\circ} \mathrm{C}$ for $2 \mathrm{~min}, 95^{\circ} \mathrm{C}$ for $10 \mathrm{~min}, 40$ cycles of $95^{\circ} \mathrm{C}$ for $15 \mathrm{~s}, 60^{\circ} \mathrm{C}$ for $1 \mathrm{~min}$. The mRNA expressions of target genes were calculated by using the $2^{-\Delta \Delta \mathrm{CT}}$ method.

\section{miRNA in situ Hybridization (ISH) Analysis}

ISH assay was performed using miRCURY locked nucleic acid (LNA) detection probe for miRNA-532-5p (Vedbaek, Denmark). Five hundred nanomoles per liter of digoxigenin double-labeled LNA-modified miR-532-5p probe was added to the hybridization solution and hybridized 
according to the instructions. After the incubation of slides with 4-nitroblue-tetrazolium and incubation with nuclear fast red (Roche Applied Science, Indianapolis, IN, USA), the images were captured under inverted microscope (Olympus, Tokyo, Japan).

\section{Western Blotting}

Total protein extracts were separated from HCCLM3 and MHCC $97 \mathrm{H}$ cells with RIPA buffer. Next, $30 \mu \mathrm{g}$ of protein extract was loaded onto $10 \%$ SDS-PAGE and transferred onto PVDF membranes. The membranes were incubated with CXCL2 (1:1000, Abcam) or GAPDH (1:1000, Abcam) antibody at $4{ }^{\circ} \mathrm{C}$ overnight and incubated with a secondary antibody $(1: 10,000$, Bioworld, Nanjing, Jiangsu, China) for $1.5 \mathrm{~h}$ at room temperature. GAPDH was used as a loading control. The band was visualized using ECL chemiluminescence (Millipore).

\section{Luciferase Reporter Assay}

The wild type (wt) or mutant type (mt) 3'-untranslated region (3'-UTR) CXCL2 was inserted into downstream of the firefly luciferase gene in the pmirGLO Dual-Luciferase miRNA Target Expression Vector (Promega, Madison, WI, USA). HCCLM3 or MHCC97H cells were seeded in 24-well plates and were cotransfected with pmirGLO vector combination with miR-532-5p by using Lipofectamine 3000 (Thermo Fisher Scientific). After $48 \mathrm{hrs}$, the luciferase activities were detected using a SpectraMax i3x microplate reader (Molecular Devices, USA).

\section{Tumor Growth of MHCC97H Cells in vivo}

MHCC97H cells $\left(5 \times 10^{6}\right)$ were implanted into BALB/C nude mice (Shanghai SLAC Laboratory Animal Center, Shanghai, China) through subcutaneous inoculation. When tumors grew to $100 \mathrm{~mm}^{3}$, the mice were randomized $(\mathrm{n}=6$ in each group) and oral gavage (p.o.) treated with vehicle control $(1 \%, \mathrm{v} / \mathrm{v}, \mathrm{DMSO}$ in normal saline) or tripterine at a dose of $10 \mathrm{mg} / \mathrm{kg}$ twice a week. After 35 days, tumor tissues were collected and subjected for immunohistochemistry (IHC) staining using CXCL2 antibody (Cell Signaling Technology). Tumor volume (TV) was calculated weekly. TV $=0.5 \times(\text { width })^{2} \times($ length $)$. The animal studies were approved by the Institutional Animal Care and Use Committee of Zhangjiagang Hospital of Traditional Chinese Medicine affiliated to Nanjing University of Chinese Medicine and was conducted in accordance with Institutional Guidelines and the Guide for the Care and Use of Laboratory Animals (NIH publication no. 85-23, revised 1996).

\section{IHC Staining}

HCC tissues or tumor tissues were fixed, dehydrated, and then subjected to IHC staining assay using Two-Step Histostaining reagent (ZSGB-BIO, Beijing, China) according to the instructions. Tissue sections were incubated with a rabbit polyclonal antibody against CXCL2 (1:200 dilution; Cell Signaling Technology). After incubation with horseradish peroxidase (HRP)-labeled goat IgG fraction to rabbit IgG $\mathrm{Fc}$, the target protein was measured using diaminobenzidine (DAB) (ZSGB-BIO). Finally, the slides were counterstained with hematoxylin and visualized under inverted microscope (Olympus).

\section{In vivo Metastasis Assay}

BALB/C nude mice (4-5 weeks) (Shanghai SLAC Laboratory Animal Center, Shanghai, China) were used for the animal studies and housed under standard conditions. MHCC97H cells $\left(5 \times 10^{7}\right)$ were intravenously injected into mice via the tail vein. The mice were divided into two groups, control group and tripterine treated group (6 per group). Mice were oral gavage (p.o.) treated with vehicle control $(1 \%, \mathrm{v} / \mathrm{v}$, DMSO in normal saline) or tripterine at a dose of $10 \mathrm{mg} / \mathrm{kg}$ twice a week. Six weeks later, the mice were sacrificed. The lungs were excised, fixed with formaldehyde solution and embedded in paraffin. The paraffin-fixed tissues were cut into $5-\mu \mathrm{m}$ sections and subjected for hematoxylin-eosin (H\&E) kit (cat \# ab245880, Abcam) staining according to the instructions. The animal studies were approved by the Institutional Animal Care and Use Committee of Zhangiiagang Hospital of Traditional Chinese Medicine affiliated to Nanjing University of Chinese Medicine and was conducted in accordance with Institutional Guidelines and the Guide for the Care and Use of Laboratory Animals (NIH publication no. 85-23, revised 1996).

\section{Statistical Analysis}

Data were expressed as mean \pm standard deviation (SD). The statistical analyses were performed using GraphPad Prism 8.0 (GraphPad Inc., San Diego, CA, USA). Differences were calculated using Student's $t$-test or one-way analysis of variance (ANOVA) followed by post hoc Dunnett's test. The relationship between the level of miR-532-5p and the mRNA level of CXCL2 was assessed by Spearman correlation analysis. $P<0.05$ indicated a significant difference. 


\section{Result}

\section{Tripterine Inhibits the}

\section{Metastatic-Associated Traits of HCC}

\section{Cells}

Before investigating the impact of tripterine (Figure 1A) on

HCC cells, CCK- 8 assay was carried out in HCC cells. The

IC50 value of tripterine in HCCLM3 and MHCC97H cells was $1.17 \mu \mathrm{M}$ and $0.96 \mu \mathrm{M}$, respectively (Supplementary

Figure 1). Considering that the IC50 value was very close to $1 \mu \mathrm{M}, 0.3,1$ and $3 \mu \mathrm{M}$ tripterine was chosen for subsequently experiments. As demonstrated in Figure 1B, tripterine $(0.3 \sim 1 \mu \mathrm{M})$ had significantly suppressive impact on HCC cells growth. We then explored whether tripterine inhibited the colony formation, migration ability and invasion capacity of HCCLM3 and MHCC97H cells in vitro. We observed that tripterine significantly decreased the colony formation of both HCCLM3 and MHCC97H cells (Figure 1C). HCCLM3 and MHCC97H cells were treated with tripterine and the migration assay was carried out. As shown in Figure 1D, the wound width was larger in higher concentration of tripterine treated HCC cells. Consistently, we observed that tripterine restrained the invasion capacity of HCCLM3 and MHCC97H cells (Figure 1E). All these findings suggested that tripterine impeded the metastaticassociated traits of HCC cells in vitro.

\section{miR-532-5p Is a Positively Regulated by Tripterine}

MiRNAs are increasingly implicated in regulating the malignant progression of cancer, including cell proliferation, migration and invasion. ${ }^{22}$ To find the potential miRNAs required for the anti-cancer activities of tripterine

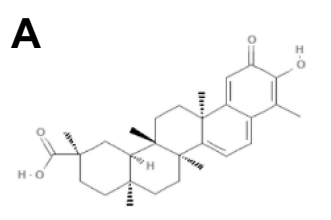

B
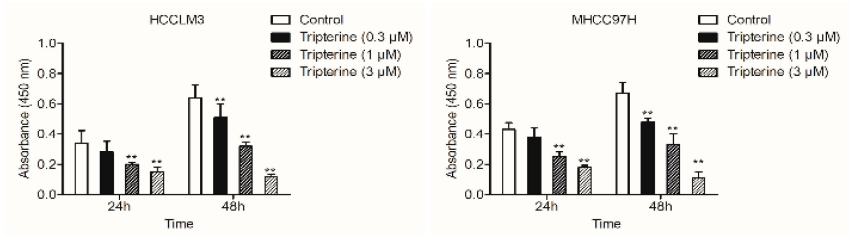

C
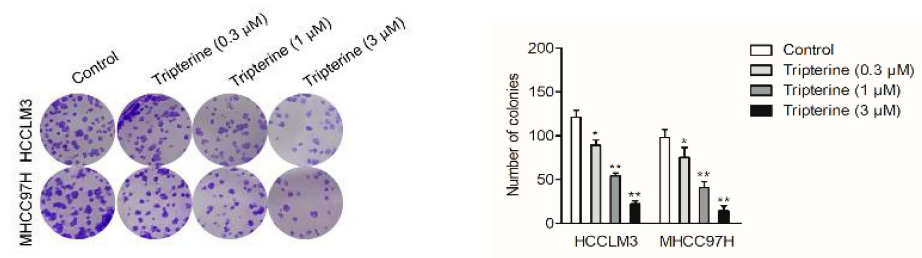

D
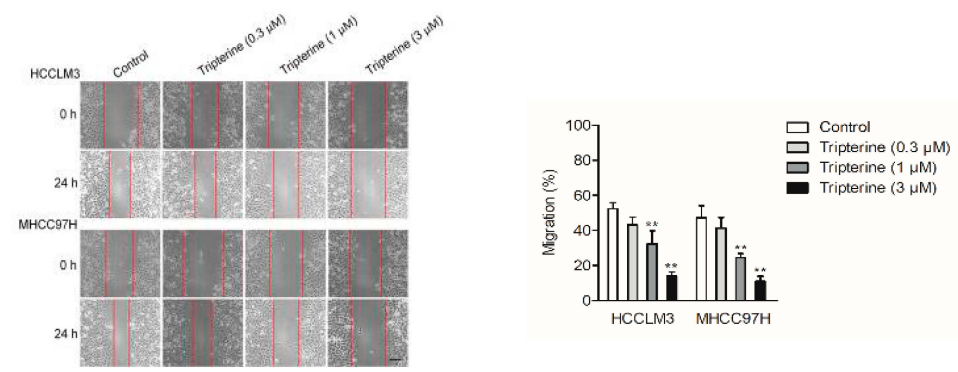

$\mathbf{E}$
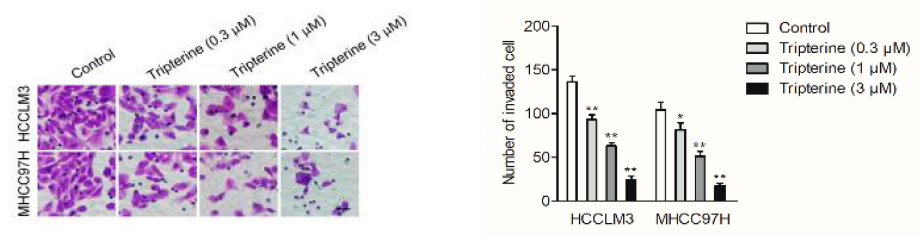

Figure I Tripterine inhibits the metastatic-associated traits of HCC cells. (A) Chemical structure of tripterine. (B) CCK-8 proliferation assay of HCCLM3 and MHCC97H cells treated with different concentrations of tripterine for $24 \mathrm{hrs}$ or $48 \mathrm{hrs}$. (C) Colony formation assay of HCCLM3 and MHCC97H cells treated with different concentrations of tripterine. (D) Wound scratch assay of HCCLM3 and MHCC97H cells treated with tripterine for 24 hrs. (E) Transwell invasion assays showed that tripterine decreased the invasion ability of HCCLM3 and MHCC $97 \mathrm{H}$ cells. Scale bar: $100 \mu \mathrm{m}$. $* P<0.05$, $* * P<0.01$ compared with control. 
in HCC, microarray was carried out to explore the miRNAs expressions regulated by tripterine. Considering that the IC50 value of tripterine in HCCLM3 and MHCC97H cells were $1.17 \mu \mathrm{M}$ and $0.96 \mu \mathrm{M}, 1 \mu \mathrm{M}$ tripterine was chosen for the subsequently microarray assay. Several miRNAs expressions were substantially altered in HCCLM3 and MHCC97H cells treated with tripterine $(1 \mu \mathrm{M})$ for $48 \mathrm{hrs}$. The dysregulation of miRNAs $(P<0.05, \log \mathrm{Fc}<-2$ or $>2)$ in HCCLM3 and MHCC97H cells treated with tripterine $(1 \mu \mathrm{M})$ were summarized in Supplementary Table 2 and Supplementary Table 3. Among the screened miRNAs, miR-532-5p was over-regulated after tripterine interference (Figure 2A). The results of qRT-PCR assays further confirmed that treatment with tripterine $(1 \mu \mathrm{M})$ remarkedly raised the expressions of miR-532-5p in both HCCLM3 and
MHCC97H cells (Figure 2B). In addition, the results from qRT-PCR also suggested that miR-532-5p was downexpressed in HCC cells compared to that in LO2 cell (Figure 2C). Thus, we suspected that miR-532-5p is positively regulated by tripterine.

\section{Tripterine Regulates the Metastatic-Related Traits of HCC Cells via Modulating miR-532-5p}

To explore the relationship between tripterine, miR-532-5p and HCC cells metastasis, HCCLM3 or MHCC97H cells were transfected with miR-532-5p inhibitor or miR-532-5p mimics. The results of qRT-PCR indicated that miR-532-5p level was markedly raised after transfection of miR-532-5p. However, the level of miR-532-5p was significantly reduced by miR-532-5p inhibitor treatment (Figure $3 \mathrm{~A}$ ). The wound

\section{A}

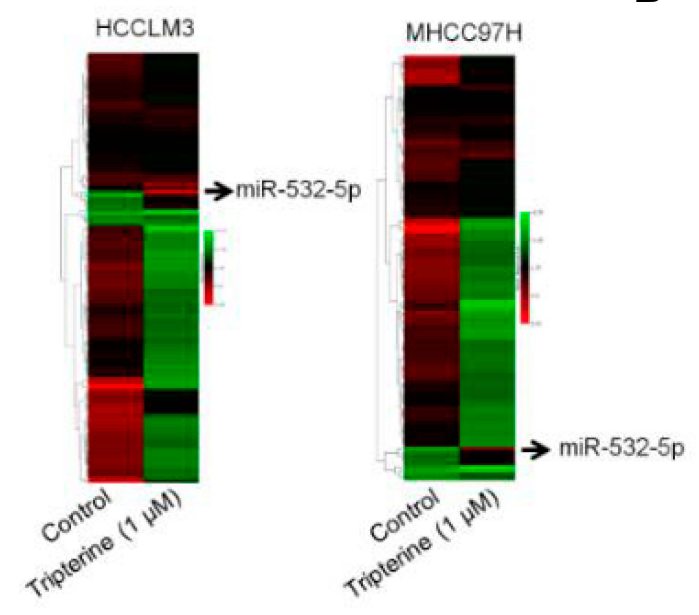

C
B

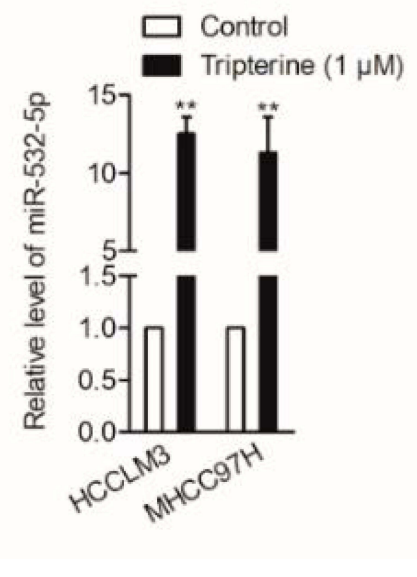

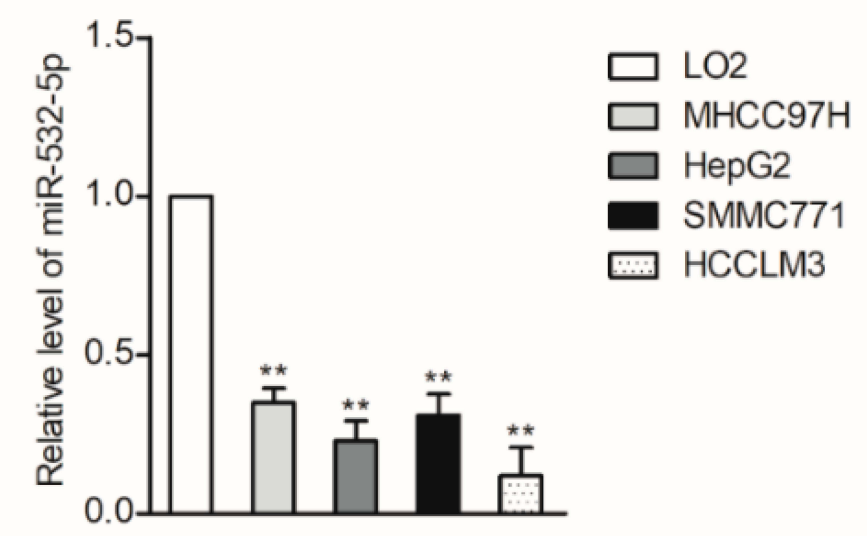

Figure 2 MiR-532-5p is negatively regulated by tripterine. (A) Heat map of the differential expression of screened miRNAs in HCCLM3 and MHCC97H cells treated or not treated with tripterine for 48 hrs. (B) The expressions of miR-532-5p in HCCLM3 and MHCC97H cells treated or not treated with tripterine for 48 hrs were detected using qRT-PCR assay. (C) miR-532-5p expression in HCC cells lines (HCCLM3, HepG2, SMMC77I and MHCC97H) and LO2 cell as determined by qRT-PCR. **P<0.0I compared with LO2 cells. 
A

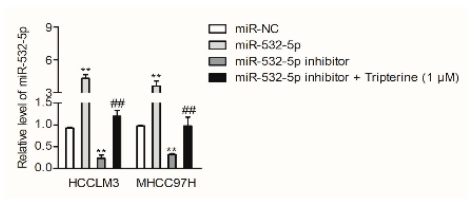

B

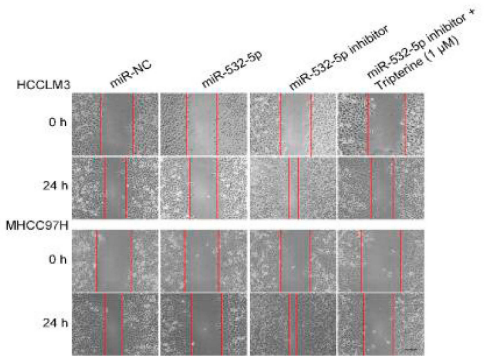

C
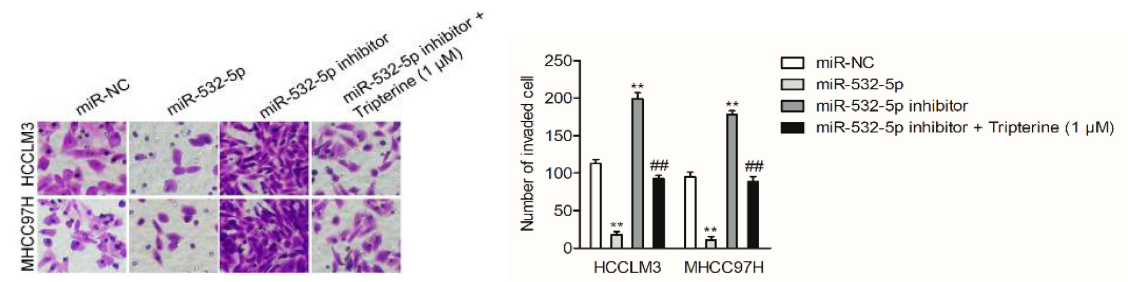

D

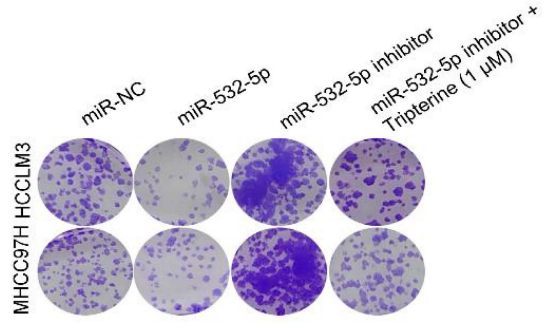

F

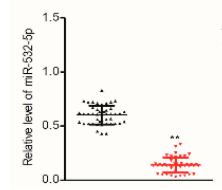

E

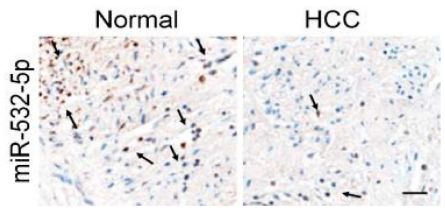

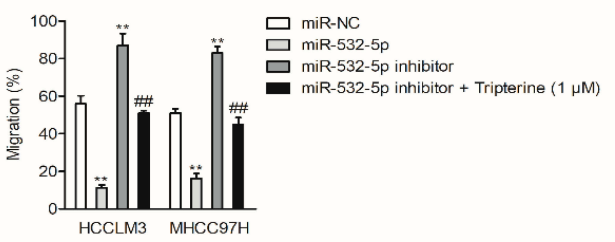

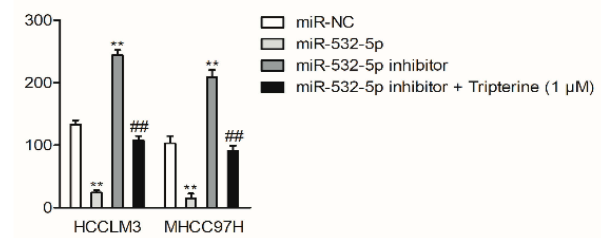

H
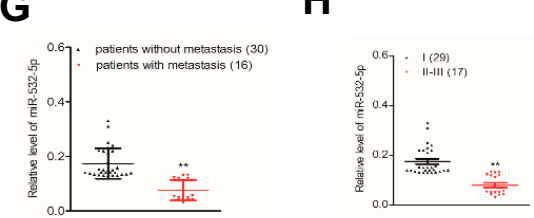

Figure 3 Inhibition of miR-532-5p is required for the metastasis of HCC cells. (A) miR-532-5p expressions in miR-532-5p inhibitor transfected cells or tripterine-treated HCCLM3 and MHCC97H cells were determined by qRT-PCR. (B) Wound healing migration assay using HCCLM3 and MHCC97H cells. (C) Transwell invasion assay. (D) Colony formation assay showed that the growth of HCCLM3 and MHCC97H cells was decreased after miR-532-5p transfection but was increased in miR-532-5p inhibitortreated cells. (E) ISH analysis of the level of miR-532-5p in HCC tissue and corresponding normal tissue. The black arrow was an indicator for miR-532-5p staining in HCC and normal tissues. (F) The levels of miR-532-5p in HCC tissues and corresponding normal tissues were measured using qRT-PCR assay. (G) The level of miR-532-5p was negatively associated with the metastasis of patients with HCC. $(\mathbf{H})$ The level of miR-532-5p was negatively associated with advance stage of HCC. Scale bar: I00 $\mu$ m. $* * P<0.01$ compared with miR-NC, \#P<0.01 compared with miR-532-5p inhibitor.

healing and Transwell invasion assays suggested that transfection of miR-532-5p distinctly decreased the migration capacity and invasiveness of HCCLM3 and MHCC97H cells whereas after treatment with miR-532-5p inhibitor, the migration and invasion abilities were enhanced (Figure 3B and C). In the presence of miR-532-5p inhibitor, the suppressive effects of tripterine on HCCLM3 and MHCC97H cells migration and invasion were weakened (Figure 3B and C). Consistently, the colony formation of HCCLM3 and MHCC97H cells were distinctly reduced by miR-532-5p and increased by miR$532-5 p$ inhibitor. In the presence of miR-532-5p inhibitor, the suppressive effects of tripterine on HCCLM3 and MHCC97H cells growth were impaired (Figure 3D). In situ hybridization (ISH) analysis indicated the miR-532-5p was down-regulated in HCC tissue (Figure 3E). MiR-532-5p level was also measured in HCC tissues by using qRT-PCR assay. 
As showed in Figure 3F, miR-532-5p was distinctly downexpressed in clinical HCC tissue when compared with that in the corresponding tissue. Finally, we found that miR-532-5p level was negatively related with clinical stage and metastasis of patients with $\mathrm{HCC}$ (Figure $3 \mathrm{G}$ and $\mathrm{H}$ ). In conclusion, tripterine regulates the metastatic-related traits of HCCLM3 and MHCC97H cells via modulating miR-532-5p.

\section{CXCL2 Is a Downstream Target of miR-532-5p}

With the help of miRNAs target prediction tools (TargetScan Release 7.1 and miRTarBase), we predicted that total five potential genes (EPC1, ZFHX3, NKD1, MDH2 and CXCL2) could be the potential targets of miR-532-5p (Figure 4A and Supplementary Table 3). The qRT-PCR assay indicted that only the expression of CXCL2 was significantly downregulated in both HCCLM3 and MHCC97H cells transfected with miR-532-5p (Figure 4B). Then, the result of luciferase reporter gene test suggested that miR-532-5p transfections remarkably reduced the luciferase activity in $\mathrm{HCC}$ cells that were transfected with wt-CXCL2 (Figure 4C). In addition,
Western blotting assay indicated that the expression of CXCL2 was reduced by miR-532-5p (Figure 4D). To investigate the association between CXCL2 and miR-532-5p in HCC, the level of CXCL2 was measured in HCC tissues using qRT-PCR analysis and IHC assay. As showed in Figure 4E and F, CXCL2 was significantly overexpressed in clinical HCC tissue compared to corresponding tissue. Finally, we observed that the expression of miR-532-5p was inversely associated with CXCL2 mRNA level in HCC tissue (Figure 4G). All these observations indicate that CXCL2 is a target of miR-532-5p in HCC and CXCL2 exerts cancer promoting roles.

\section{miR-532-5p Targets CXCL2 to Modulate the Metastasis of HCC Cells}

To elaborate the underlying mechanisms of miR-532-5p exerted its effect on HCC cells, HCCLM3 and MHCC97H cells were cotransfected with miR-532-5p with pc-CXCL2. Next, the qRT-PCR and Western blotting analysis demonstrated that reexpression of CXCL2 recovered the expression of CXCL2 in HCCLM3 and MHCC97H cells that was
A

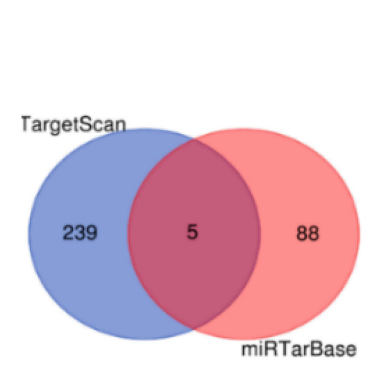

B

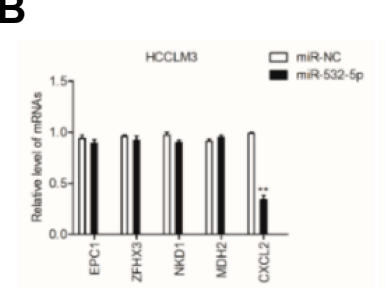

E

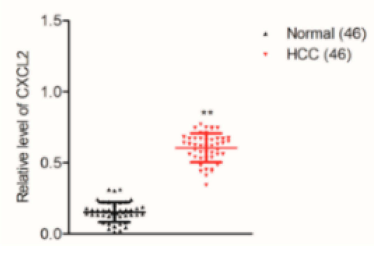

\section{C}

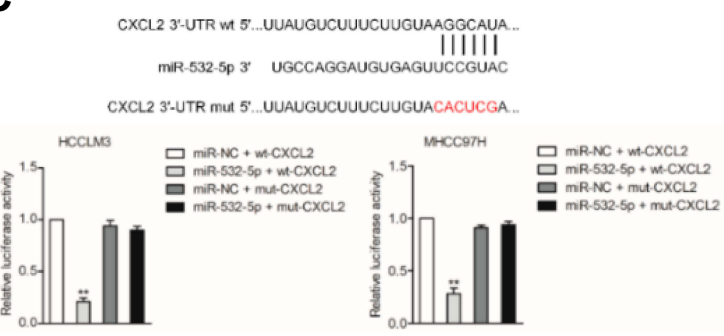

D

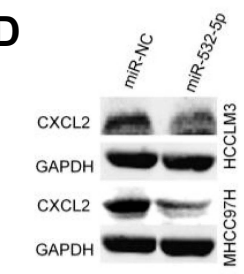

G

$\mathbf{F}$
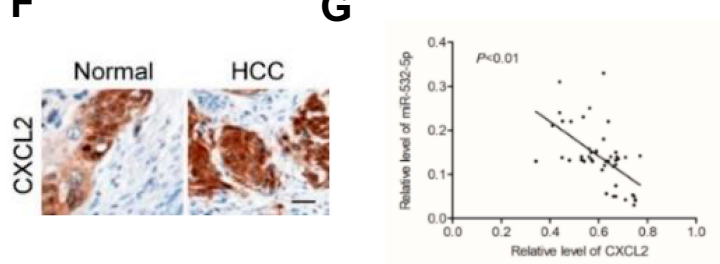

Figure 4 CXCL2 is a target gene of miR-532-5p. (A) Venn diagram depicts a common target gene in TargetScan Release 7.I and miRTarBase.(B) The mRNA levels of potential target genes (EPCI, ZFHX3, NKDI, MDH2 and CXCL2) in HCCLM3 and MHCC97H cells transfected with miR-532-5p were measured using qRT-PCR assay. (C) The interaction between CXCL2 and miR-532-5p was predicted by TargetScan. miR-532-5p overexpression reduced the luciferase activity of vector carrying wt CXCL2 rather than mut CXCL2 in HCCLM3 and MHCC97H cells. (D) The expression of CXCL2 in cell that was transfected with miR-532-5p was detected using immunofluorescence analysis. (E) The level of CXCL2 in HCC tissue was measured using qRT-PCR analysis. (F) The expression on CXCL2 was measured using IHC staining. (G) The level of miR-532-5p was inversely associated with CXCL2 level in HCC tissue. Scale bar: $100 \mu m$. $* * P<0.01$ compared with miR-NC. 
transfected with miR-532-5p (Figure 5A and B). In addition, overexpression of CXCL2 rescued the proliferation, colony formation ability, migration and invasiveness of HCCLM3 and MHCC97H cells inhibited by miR-532-5p (Figure 5C-F). Altogether, $\mathrm{CXC} 2$ is a functional downstream target of miR-532-5p and is negatively regulated by miR-532-5p in both HCCLM3 and MHCC97H cells.

\section{Tripterine Inhibits the Metastasis of HCC Cells via Regulating miR-532-5p-CXCL2}

\section{Axis}

To investigate the underlying mechanism of tripterine exerted its effect on HCC cells, HCCLM3 and MHCC97H cells were transfected with pc-CXCL2 in the presence of tripterine. The qRT-PCR and Western blotting analysis demonstrated that tripterine-inhibited CXCL2 expression in HCCLM3 and MHCC97H cells whereas the level of CXCL2 was restored by pc-CXCL2 transfection (Figure 6A and $\mathrm{B}$ ). In addition, the colony formation, migration and invasion abilities of HCCLM3 and MHCC97H cells inhibited by tripterine were rescued by transfection of pc-CXCL2 (Figure 6C-E). In summary, we conclude that tripterine inhibits the metastatic abilities of HCC cells via regulating miR-532-5p-CXCL2 axis.

\section{Tripterine Suppresses the Growth of HCC Cells in vivo}

To explore the relationship between tripterine and HCC cells growth in vivo, MHCC97H cells were subcutaneously inoculated into BALA/c nude mice and then

A

C
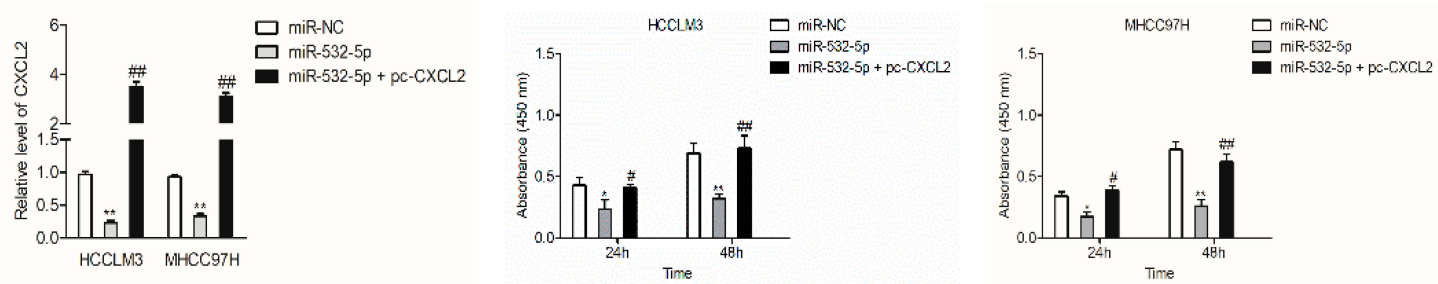

B

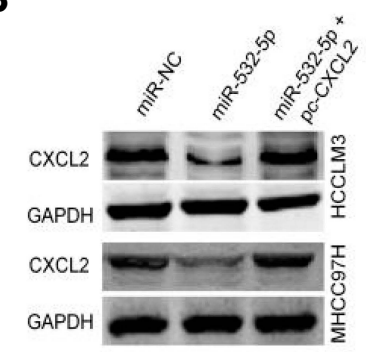

D
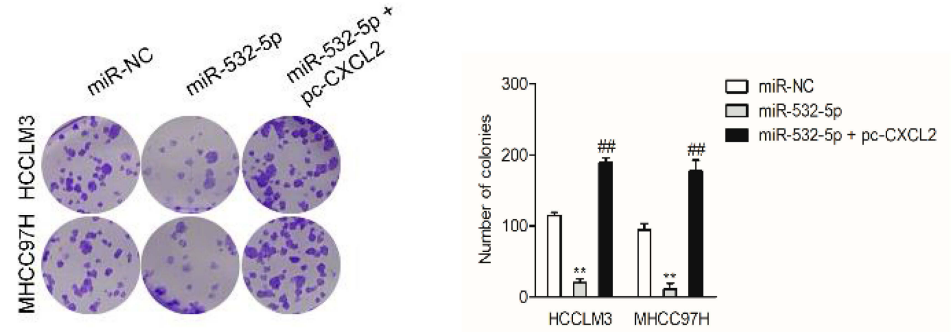

E

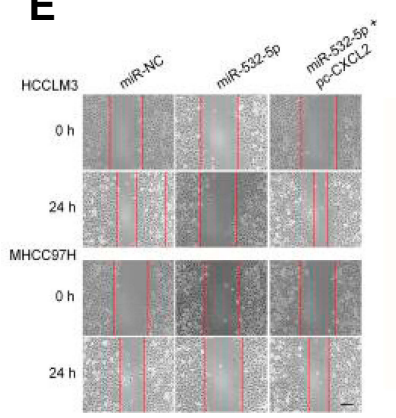

$\mathbf{F}$

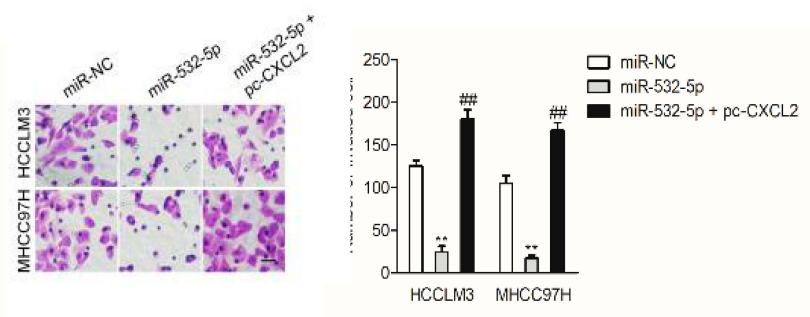

Figure 5 MiR-532-5p directly targets CXCL2 to mediate the metastasis of HCC cells. (A, B) HCCLM3 and MHCC97H cells were cotransfected with miR-532-5p and pcCXCL2. The expression of CXC2 was detected using qRT-PCR and immunofluorescence analysis. (C-F) HCCLM3 and MHCC97H cells were cotransfected with miR-532$5 p$ and $p c-C X C L 2$. The proliferation, colony formation, migration and invasion of HCCLM3 and MHCC97H cells were analyzed. Scale bar: $100 \mu m$. $* P<0.05$ and $* * P<0.01$ compared with miR-NC, \#P<0.05 and \#\#P<0.01 compared with miR-532-5p. 
A
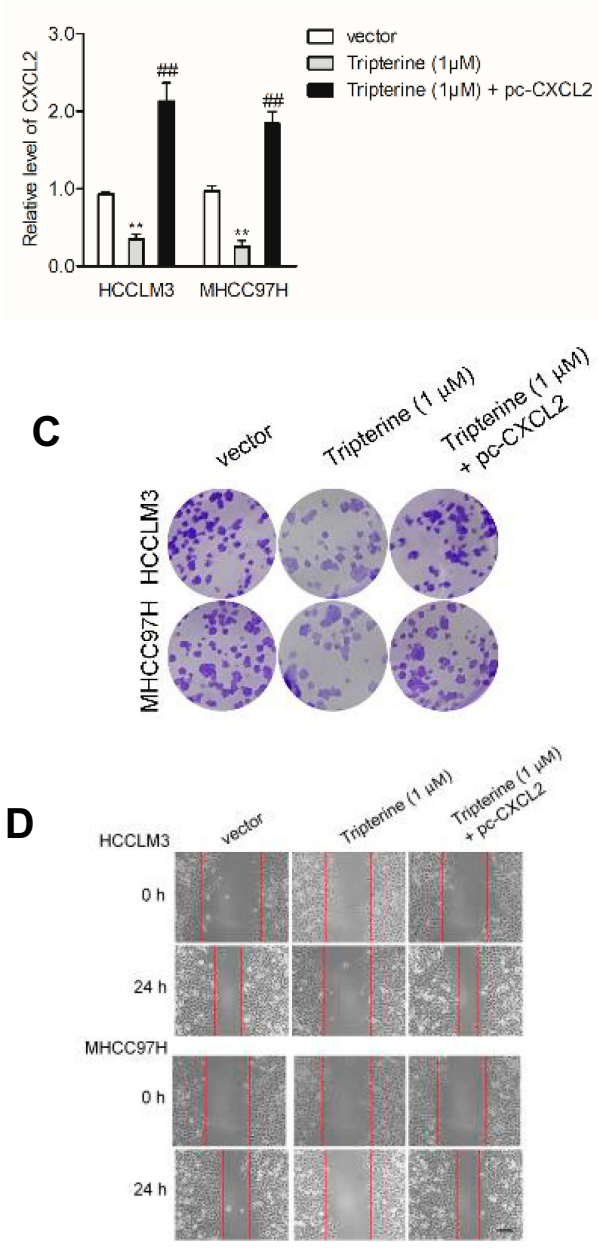

$\mathbf{E}$

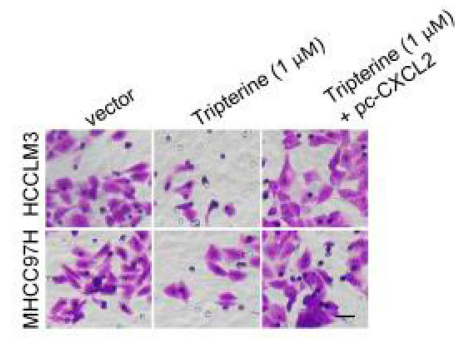

B
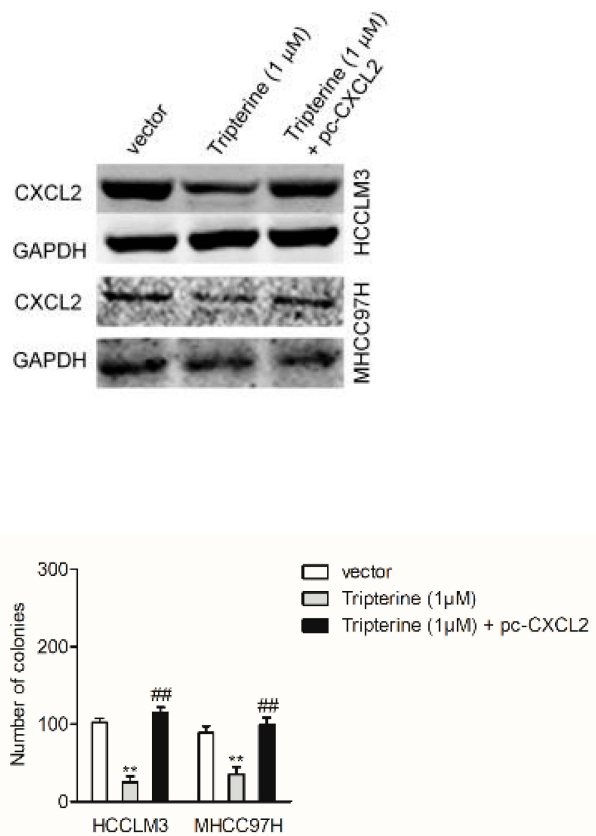

Figure 6 Tripterine mediate the metastasis of $\mathrm{HCC}$ cells via regulating CXCL2. (A, B) HCCLM3 and MHCC97H cells were transfected with pc-CXCL2 in the presence of tripterine. The expression of $\mathrm{CXC2}$ was detected using qRT-PCR and immunofluorescence analysis. (C-E) HCCLM3 and MHCC97H cells were transfected with pc-CXCL2 in the presence of tripterine. The colony formation, migration and invasion of HCCLM3 and MHCC97H cells were analyzed. Scale bar: $100 \mu m$. $* * P<0.01$ compared with vector, \#\#P<0.0I compared with tripterine $(I \mu M)$.

mice were treated with tripterine. We found that treatment with tripterine repressed the tumor growth of MHCC97H cells in vivo (Figure 7A-C). Meanwhile, the primary tumors were stained using CXCL2 antibody, and we observed that the level of CXCL2 was lower in mice that are treated with tripterine compared to vehicle-treated mice (Figure 7D). As expected, the level of miR-532-5p in mice treated with tripterine was significantly increased compared to vehicle-treated mice (Figure 7E). Finally, experimental lung metastasis model was constructed to investigate the inhibitory role of tripterine on distant metastasis ability of MHCC97H cells in vivo. More pulmonary metastatic foci occurred in the control group whereas considerably lower number and size of pulmonary 


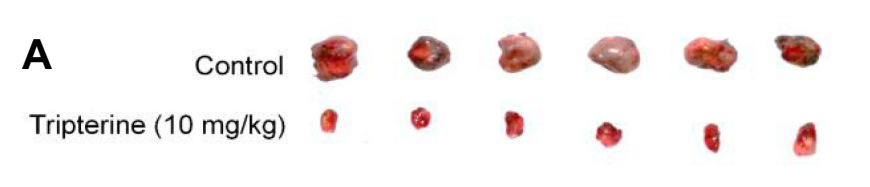

B
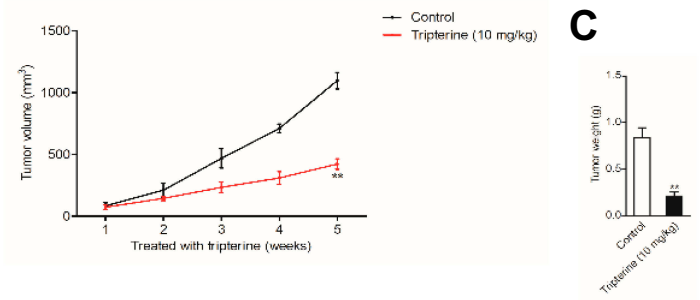

D

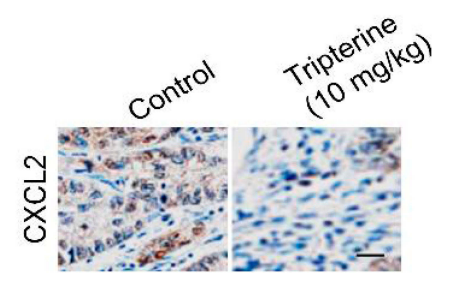

E

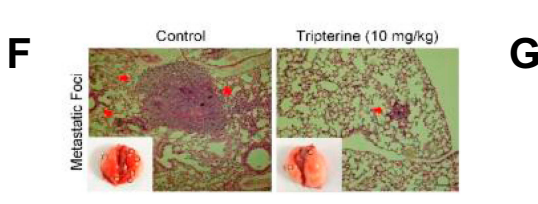

$\mathrm{PI} 3 \mathrm{~K} / \mathrm{AKT} / \mathrm{NF}-\kappa \mathrm{B}$ signaling axis. $^{26}$ Although several investigations have reported the anti-cancer effects of tripterine in malignant tumors, no investigation has been reported on the anti-metastatic roles of tripterine in $\mathrm{HCC}$. Herein, we explored the impact of tripterine on growth, migration and invasion abilities of HCCLM3 and MHCC97H cells in vitro. Besides, tripterine efficiently raised the level of miR-532-5p in HCC cells. Furthermore, over-regulation of miR-224 expression inhibited CXCL2 level. So, we speculate that the impacts of tripterine on suppression of migration and invasion abilities of $\mathrm{HCC}$ cells were probably due to the intervening of miR-532-5pCXCL2. We further found that CXCL2 overexpression reversed the impacts of tripterine on inhibition migration and invasion capacities of HCC cells.

Previous investigations have suggested that the alterations in the expressions of miRNAs are related with the progression and clinical outcomes of cancers. Previous studies have suggested that the levels of miRNA-532-5p are higher in breast, stomach, liver and cervical cancer tissues, which indicating that miRNA-532-5p may act as suppressor in tumorigenesis. For instance, miR-532-5p is a valuable prognostic indicator and restrains the growth and aggressiveness of epithelial ovarian cancer cell via regulating Twist Family BHLH Transcription Factor 1 (TWIST1). ${ }^{27}$ Downexpression of miRNA-532-5p induces the growth and aggressive ability of bladder cancer cell via intervening High Mobility Group Box 3 (HMGB3) signaling. ${ }^{28}$ In this study, we validated that miR-532-5p regulates the metastatic-related traits of $\mathrm{HCC}$ cells. Upregulation of miR-532-5p significantly decreased the growth, colony formation ability, migration and invasiveness of HCC cells. To investigate the potential functions of tripterine in anti-cancer, HCC cells were treated with tripterine. The data indicated that tripterine inhibited the metastasis of HCC cells. Nevertheless, when downexpressing miR-532-5p cells were treated with tripterine, migration ability and invasiveness were diminished, which proved that tripterine suppresses metastasis by increasing miR-532-5p.

MiRNAs are critical regulators among diverse pathological processes via directly binding with the 3 '-UTR of mRNAs and serve as tumor suppressors or oncogenes. ${ }^{29-31}$ Here, we identified CXCL2 as a downstream target gene of miR-532-5p. The level of CXCL2 was negatively associated with miR-532-5p level in HCC and its level was negatively modulated by miR-532-5p. Importantly, upregulation of CXCL2 restored the migration ability and 
invasive capacity of HCC cells suppressed by miR-532-5p, which indicating that CXCL2 was a functional target gene of miR-532-5p. Next, CXCL2 overexpressing HCC cells were treated with tripterine to investigate the underlying mechanism of tripterine exerted its effect on HCC cells. As expected, overexpression of CXCL2 rescued the aggressiveness of HCC cells, which indicates that tripterine inhibits HCC cells metastasis via regulating miR-532$5 \mathrm{p}-\mathrm{CXCL} 2$ axis.

Finally, the growth of HCC cells in vivo was also significantly restrained by tripterine. The expression of CXCL2 was distinctly decreased and miR-532-5p level was increased by tripterine in vivo. All these observations indicate that inhibition of the miR-532-5p/CXCL2 pathway is involved into tripterine-inhibited HCC cells growth and metastasis.

\section{Conclusions}

Our present study demonstrates that tripterine increases the level of miR-532-5p, which in turn reduces CXCL2 expression in HCC. Overregulation of miR-532-5p expression results in the decrease of CXCL2 level as well as inhibition of metastatic-related phenotypes of HCC cells. All these findings imply that tripterine restrains the progression of hepatocellular carcinoma cell by regulating miRNA-532-5p/CXCL2 axis.

\section{Acknowledgment}

This study was supported by Pharmaceutical research project of Tianqing (Lianyungang) Hospital of Jiangsu Pharmaceutical Association (Q2018169) and the Fifth Batch of Suzhou Health Talents Project (GSWS2019075).

\section{Disclosure}

The authors report no conflicts of interest in this work.

\section{References}

1. Ma Y, Ma M, Ma L, et al. Downregulation of miR-552 in hepatocellular carcinoma inhibits cell migration and invasion, and promotes cell apoptosis via RUNX3. Exp Ther Med. 2019;18(5):3829-3836. doi:10.3892/etm.2019.8061

2. Qi Y, Yao X, Du X. Midazolam inhibits proliferation and accelerates apoptosis of hepatocellular carcinoma cells by elevating microRNA-124$3 \mathrm{p}$ and suppressing PIM-1. IUBMB Life. 2020;72(3):452-464. doi:10.1002/iub.v72.3

3. Ke M, Zhang Z, Cong L, et al. MicroRNA-148b-colony-stimulating factor-1 signaling-induced tumor-associated macrophage infiltration promotes hepatocellular carcinoma metastasis. Biomed Pharmacother. 2019;120:109523. doi:10.1016/j.biopha.2019.109523

4. Abu Bakar MH, Sarmidi MR, Tan JS, Mohamad Rosdi MN. Celastrol attenuates mitochondrial dysfunction and inflammation in palmitate-mediated insulin resistance in C3A hepatocytes. Eur J Pharmacol. 2017;799:73-83. doi:10.1016/j.ejphar.2017.01.043
5. Kuchta K, Xiang Y, Huang S, et al. Celastrol, an active constituent of the TCM plant tripterygium wilfordii Hook.f., inhibits prostate cancer bone metastasis. Prostate Cancer Prostatic Dis. 2017;20(2):250. doi:10.1038/pcan.2017.11

6. Moreira H, Szyjka A, Gasiorowski K. Chemopreventive activity of celastrol in drug-resistant human colon carcinoma cell cultures. Oncotarget. 2018;9(30):21211-21223. doi:10.18632/oncotarget.25014

7. Guan Y, Cui ZJ, Sun B, et al. Celastrol attenuates oxidative stress in the skeletal muscle of diabetic rats by regulating the AMPK-PGC1alphaSIRT3 signaling pathway. Int J Mol Med. 2016;37(5):1229-1238. doi:10.3892/ijmm.2016.2549

8. Liu J, Lee J, Salazar Hernandez MA, Mazitschek R, Ozcan U. Treatment of obesity with celastrol. Cell. 2015;161(5):999-1011. doi:10.1016/j.cell.2015.05.011

9. Venkatesha SH, Dudics S, Astry B, Moudgil KD, Flajnik M. Control of autoimmune inflammation by celastrol, a natural triterpenoid. Pathog Dis. 2016;74(6):ftw059. doi:10.1093/femspd/ftw059

10. Venkatesha SH, Moudgil KD. Celastrol and its role in controlling chronic diseases. Adv Exp Med Biol. 2016;928:267-289.

11. Pang X, Yi Z, Zhang J, et al. Celastrol suppresses angiogenesis-mediated tumor growth through inhibition of AKT/mammalian target of rapamycin pathway. Cancer Res. 2010;70(5):1951-1959. doi:10.1158/00085472.CAN-09-3201

12. He Z, Yi J, Liu X, et al. MiR-143-3p functions as a tumor suppressor by regulating cell proliferation, invasion and epithelial-mesenchymal transition by targeting QKI-5 in esophageal squamous cell carcinoma. Mol Cancer. 2016;15(1):51. doi:10.1186/s12943-0160533-3

13. Fang Z, Yin S, Sun R, et al. miR-140-5p suppresses the proliferation, migration and invasion of gastric cancer by regulating YES1. Mol Cancer. 2017;16(1):139. doi:10.1186/s12943-017-0708-6

14. Zhai W, Ma J, Zhu R, et al. MiR-532-5p suppresses renal cancer cell proliferation by disrupting the ETS1-mediated positive feedback loop with the KRAS-NAP1L1/P-ERK axis. Br J Cancer. 2018;119 (5):591-604. doi:10.1038/s41416-018-0196-5

15. Song X, Wang Z, Jin Y, Wang Y, Duan W. Loss of miR-532-5p in vitro promotes cell proliferation and metastasis by influencing CXCL2 expression in HCC. Am J Transl Res. 2015;7 (11):2254-2261.

16. Islam J, Koseki T, Watanabe K, et al. Dietary supplementation of fermented rice bran effectively alleviates dextran sodium sulfate-induced colitis in mice. Nutrients. 2017;9(7):E747. doi:10.3390/nu9070747

17. Zhou W, Zhang C, Jiang H, et al. MiR-493 suppresses the proliferation and invasion of gastric cancer cells by targeting RhoC. Iran J Basic Med Sci. 2015;18(10):1027-1033.

18. Sikand K, Singh J, Ebron JS, Shukla GC, Stoecklin G. Housekeeping gene selection advisory: glyceraldehyde-3-phosphate dehydrogenase (GAPDH) and beta-actin are targets of miR-644a. PLoS One. 2012;7 (10):e47510. doi:10.1371/journal.pone.0047510

19. Ma J, Zhang J, Wang Y, et al. MiR-532-5p alleviates hypoxia-induced cardiomyocyte apoptosis by targeting PDCD4. Gene. 2018;675:36-43. doi:10.1016/j.gene.2018.06.087

20. Lou G, Ma N, Xu Y, et al. Differential distribution of U6 (RNU6-1) expression in human carcinoma tissues demonstrates the requirement for caution in the internal control gene selection for microRNA quantification. Int J Mol Med. 2015;36(5):1400-1408. doi:10.3892/ ijmm.2015.2338

21. Daniel R, Wu Q, Williams V, et al. A panel of MicroRNAs as diagnostic biomarkers for the identification of prostate cancer. Int J Mol Sci. 2017;18(6):E1281. doi:10.3390/ijms18061281

22. Ma L, Young J, Prabhala H, et al. miR-9, a MYC/MYCN-activated microRNA, regulates E-cadherin and cancer metastasis. Nat Cell Biol. 2010;12(3):247-256. doi:10.1038/ncb2024

23. Chen Y, Qu D, Fu R, et al. A Tf-modified tripterine-loaded coix seed oil microemulsion enhances anti-cervical cancer treatment. Int J Nanomedicine. 2018;13:7275-7287. doi:10.2147/IJN 
24. Zuo A, Zhao P, Zheng Y, Hua H, Wang X. Tripterine inhibits proliferation, migration and invasion of breast cancer MDA-MB231 cells by up-regulating microRNA-15a. Biol Chem. 2019;400 (8):965-1086. doi:10.1515/hsz-2018-0469

25. Yao SS, Han L, Tian ZB, et al. Celastrol inhibits growth and metastasis of human gastric cancer cell MKN45 by down-regulating microRNA-21. Phytother Res. 2019;33(6):1706-1716. doi:10.1002/ptr.6359

26. Zhang H, Li J, Li G, Wang S. Effects of celastrol on enhancing apoptosis of ovarian cancer cells via the downregulation of microRNA21 and the suppression of the PI3K/AktNFkappaB signaling pathway in an in vitro model of ovarian carcinoma. Mol Med Rep. 2016;14(6):5363-5368. doi:10.3892/mmr.2016.5894

27. Wei H, Tang QL, Zhang K, Sun JJ, Ding RF. miR-532-5p is a prognostic marker and suppresses cells proliferation and invasion by targeting TWIST1 in epithelial ovarian cancer. Eur Rev Med Pharmacol Sci. 2018;22(18):5842-5850. doi:10.26355/eurrev 20180915911
28. Xie X, Pan J, Han X, Chen W. Downregulation of microRNA-532-5p promotes the proliferation and invasion of bladder cancer cells through promotion of HMGB3/Wnt/beta-catenin signaling. Chem Biol Interact. 2019;300:73-81. doi:10.1016/j.cbi.2019.01.015

29. Yang R, Liu M, Liang $H$, et al. miR-138-5p contributes to cell proliferation and invasion by targeting survivin in bladder cancer cells. Mol Cancer. 2016;15(1):82. doi:10.1186/s12943-016-0569-4

30. Huang Y, Huang H, Li M, et al. MicroRNA-374c-5p regulates the invasion and migration of cervical cancer by acting on the Foxc1/ snail pathway. Biomed Pharmacother. 2017;94:1038-1047. doi:10.1016/j.biopha.2017.07.150

31. Shen YY, Cui JY, Yuan J, Wang X. MiR-451a suppressed cell migration and invasion in non-small cell lung cancer through targeting ATF2. Eur Rev Med Pharmacol Sci. 2018;22(17):5554-5561. doi:10.26355/eurrev_201809_15818

\section{Publish your work in this journal}

OncoTargets and Therapy is an international, peer-reviewed, open access journal focusing on the pathological basis of all cancers, potential targets for therapy and treatment protocols employed to improve the management of cancer patients. The journal also focuses on the impact of management programs and new therapeutic agents and protocols on patient perspectives such as quality of life, adherence and satisfaction. The manuscript management system is completely online and includes a very quick and fair peer-review system, which is all easy to use. Visit http://www.dovepress.com/ testimonials.php to read real quotes from published authors. 